\title{
LACK OF HYPERBOLICITY IN ASYMPTOTIC ERDÖS-RENYI SPARSE RANDOM GRAPHS
}

\author{
Onuttom Narayan, ${ }^{1}$ Iraj Saniee, ${ }^{2}$ and Gabriel H. Tucci ${ }^{2}$ \\ ${ }^{1}$ Department of Physics, University of California, Santa Cruz, California, USA \\ ${ }^{2}$ Bell Laboratories, Alcatel-Lucent, Murray Hill, New Jersey, USA
}

\begin{abstract}
In this work, we prove that the giant component of the Erdös-Renyi random graph $G(n, c / n)$ for c a constant greater than 1 (sparse regime), is not Gromov $\delta$-hyperbolic for any $\delta$ with probability tending to one as $n \rightarrow \infty$. We present numerical evidence to show that the "fat" triangles that rule out $\delta$-hyperbolicity are in fact abundant in these graphs. We also present numerical evidence showing that, despite the known fact that these graphs almost surely have zero spectral gap as $n \rightarrow \infty$, the spectrum is very similar in outline to that of an infinite regular tree with the same (average) degree, which can be calculated analytically.
\end{abstract}

\section{INTRODUCTION AND MOTIVATION}

Random graphs constitute an important and active research area with numerous applications to geometry, percolation theory, information theory, queuing systems, and communication networks, to mention a few. They also provide analytical means to settle prototypical questions and conjectures that might be harder to resolve in specific circumstances (such as statistical evidence for hyperbolicity or its lack via curvature plots, as discussed in [16], which is our focus here). In this work, we study two questions with regard to the asymptotic geometry of Erdös-Renyi random graphsErdos59,Gilbert59,Chung94, partly motivated by inference from earlier work that random graphs may be hyperbolic [12] or may have spectral gaps [6]. These and other authors use the term random graph in different senses. To fix definition and notation, we call $G\left(n, p_{n}\right)$ a random graph where $n$ is the number of nodes and $p_{n}$ is the probability of an edge between any node pair, independent of all other edges. The construction of a $G\left(n, p_{n}\right)$ consists of connecting any pair of these $n$ nodes independently, each with probability $p_{n}{ }^{1}$

Our main result is that in the constant average-degree regime $p_{n}=c / n$ with $c$ a constant greater than 1 , with probability approaching 1 , these graphs are not $\delta$-hyperbolic in the sense of Gromov [10] (which we make precise in Section 2) for any nonnegative $\delta$. One might think that this is equivalent to the lack of spectral gap, because Gromov's notion of hyperbolicity and the linear isoperimetric inequality are intimately related in a coarse

\footnotetext{
${ }^{1}$ This is actually the $G(n, p)$ model of a random graph of GilbertGilbert59 rather than the ErdösRenyiErdos59 model known as $G(n, M)$; but we follow the now almost universal proclivity of referring to these as Erdös-Renyi random graphs.

Address correspondence to Onuttom Narayan, Department of Physics, University of California, Santa Cruz, CA 95064, USA. E-mail: onarayan@ucsc.edu

Color versions of one or more of the figures in the article can be found online at www.tandfonline.com/uinm.
} 
sense; see [3]. In fact, despite the connection between the two, neither one implies the other, as we discuss in more detail in Section 2. This implies that the questions of hyperbolicity and spectral gap of random graphs need to be addressed independently.

This article is organized as follows. In Section 2, we show that the largest component of $G\left(n, p_{n}\right)$ is not $\delta$-hyperbolic for any $\delta$ with probability tending to 1 as $n \rightarrow \infty$. This implies that triangles in $G\left(n, p_{n}\right)$ are not $\delta$-thin for any $\delta>0$. In Section 3, we present plots that suggest that "fat" triangles not only exist a.s as $n \rightarrow \infty$, but are abundant in finite random graphs. We also present numerical results that show a surprising degree of closeness between the spectral distribution of the normalized Laplacian of $G(n, c / n)$ and that of $c$-regular trees using well-known explicit formulas due to [14].

\section{NONHYPERBOLICITY FOR THE ERDÖS-RENYI RANDOM GRAPHS}

\subsection{Relationship between Hyperbolicity and Spectral Gap}

It is known that the giant component of $G(n, c / n)$ does not have a spectral gap a.s. in the regime $c>1$. This follows, for instance, from $[8,1]$, to cite recent papers. This means that as $n \rightarrow \infty$, the smallest nonzero eigenvalue of the Laplacian of $G(n, c / n)$ [4] goes to zero. The proof relies on the existence of long chains in the graph, whose interior nodes are not connected to the rest of the graph.

In the present study, we prove that the existence of similar (but distinct) large loops also results in the giant component not being $\delta$-hyperbolic. Despite being based on similar graph structures, the lack of a spectral gap and the absence of $\delta$-hyperbolicity are, as discussed at the end of this subsection, independent features of these graphs; thus our results do not follow directly from [8] and [1]. In the process, we provide an elementary proof of the existence of large loops, using less machinery than used in [8] for long chains.

To be more precise about the expression " $\delta$-hyperbolic" for a family of finite graphs, let $G=(V, E)$ be a (finite) graph together with an edge metric $d$ (thus $d$ satisfies the triangle inequality). Let $[X Y]$ denote a shortest path between vertices $X$ and $Y$ in $G$. A triangle $\{X, Y, Z\}$ is said to be $\delta$-thin if

$$
[X Y] \subseteq \mathcal{N}([Y Z], \delta) \cup \mathcal{N}([Z X], \delta)
$$

where $\mathcal{N}([X Y], \delta)$ is the $\delta$ neighborhood of $[X Y]$, and similarly for $[Y Z]$ and $[X Z]$. If all its triangles are $\delta$-thin, $G$ is said to be $\delta$-hyperbolic. Intuitively, $\delta$-hyperbolicity means that any three shortest paths $[X Y],[Y Z]$, and $[Z X]$ among any triple of vertices $\{X, Y, Z\}$ in $G$ come to within a distance $\delta$ of each other for some fixed $\delta \geq 0$. Thus, trees are 0 -hyperbolic, the two-dimensional square grid is not $\delta$-hyperbolic for any finite $\delta$ but any finite graph with diameter $\Delta \geq 0$ is $\Delta$-hyperbolic.

We say a family $\left\{G_{n} ; n \geq 1\right\}$ is $\delta$-hyperbolic if each member $G_{n}$ is $\delta$-hyperbolic for a fixed value $\delta>0$ and a family is asymptotically $\delta$-hyperbolic if for a large enough $n$, all $G_{n}$ are $\delta$-hyperbolic. When a family $\left\{G_{n} ; n \geq 1\right\}$ is not $\delta$-hyperbolic for any $\delta \geq 0$, then it must be the case that for any $\delta \geq 0$ there is an $n$ such that there are some $\delta$-fat triangles in $G_{n}$. This is precisely the sense in which we prove the family $G\left(n, p_{n}=c / n\right), c>1$ is not $\delta$-hyperbolic.

Naïvely, the concept of $\delta$-hyperbolicity may be associated with the existence of a spectral gap. This is because for standard hyperbolic spaces with constant negative curvature, or more pertinently for this discussion, their skeletal representations as hyperbolic 
graphs (e.g., $q$-regular trees or the $H_{p, q}$ regular hyperbolic tessellations, which are infinite planar graphs with uniform degree $q$ and $p$-gons as faces with $(p-2)(q-2)>4)$, the two coincide.

Indeed, it might be thought that the existence of a spectral gap and $\delta$-hyperbolicity are equivalent: the first is clearly equivalent to the existence of a linear isoperimetric inequality, and the second is shown to be equivalent to a linear isoperimetric inequality in Proposition III.2.7 of [3]. However, the term linear isoperimetric inequality is used in different senses in the two cases. In the first, the entire perimeter of any arbitrary subset $S$ has to be considered. In the second, disklike subsets are considered, and only the loop part of the perimeter (ignoring any boundary edges on the "flat" part of the disk) is used. Thus, neither does the existence of a spectral gap imply $\delta$-hyperbolicity nor vice versa. ${ }^{2}$

As examples to illustrate this fact, we note that a graph that consists of an infinite chain (the integers $\mathbb{Z}$ ) has a zero Cheeger constant and a zero spectral gap, even though it is $\delta$-hyperbolic because-as discussed in the next subsection-all tree graphs trivially are. On the other hand, the Cayley graph associated with the product of two free groups, $G=\mathbb{F}_{2} \times \mathbb{F}_{2}$, has a positive Cheeger constant and a nonzero spectral gap. But because it includes the graph $H=\mathbb{Z} \times \mathbb{Z}$ (the Euclidean grid) as a subgraph, it is not hyperbolic. Thus, questions with regard to the spectral gap and hyperbolicity need to be addressed independently.

\subsection{Positive Measure of Large Loops}

It is commonly stated that the Erdös-Renyi random graphs are "treelike" for large values of $n$, on the strength of the notion that any small neighborhood (the "small scale") has a very small probability of localized links; see $[15,13]$. This "treeness" in the small scale is sometimes loosely interpreted to imply that random graphs are hyperbolic. There are several concerns about these heuristic notions and clarification is needed. First, the probability regime of the construction of the random graph needs to be specified. Second, more formal definitions of small, middle, and large scale are needed. As is well known, there are different regimes in the $G(n, p)$ model of a random graph:

1. $p=o(1 / n)$, then the random graph is a large collection of disconnected small trees.

2. $p=c / n$ with $0<c<1$, then all the connected components of the graph are either trees or unicycle components. The largest connected component is a tree and has $O(\log (n))$ nodes.

3. $p=c / n$ with $c>1$, then a giant component emerges. This one has $\gamma(c) n$ nodes a.s. where $\gamma$ is a function depending on $c$ and independent on $n$. Also, the average degree of a node is away from 0 .

4. $p=c \log (n) / n$ with $c>1$, then the graph is a.s connected.

Beyond these, for example when $p=O(1 / \log (n))$, there is a single highly connected component whose average nodal degree is unbounded as $n \rightarrow \infty$.

To define and clarify the relevant scale and the notion of hyperbolicity for random graphs, we use the definition of (coarse) hyperbolicity in the large scale [10]. The natural setting for the definition is within path-metric spaces, which for our purposes simplify to metric graphs in which a non-negative metric is defined on the links (or nodes or both) of

\footnotetext{
${ }^{2}$ We thank M. R. Bridson for a useful discussion on this point.
} 


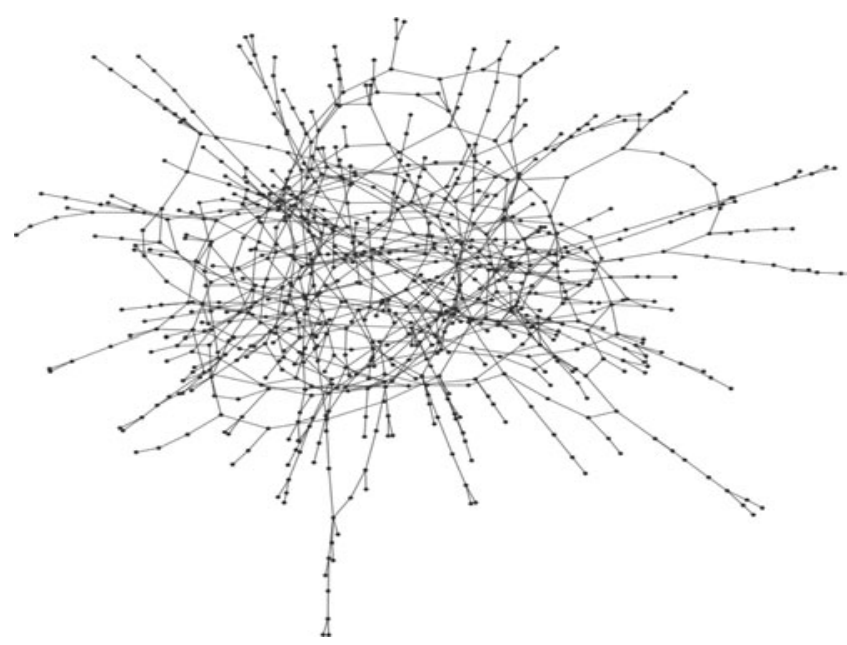

Figure 1 A random graph $G(n, 2 / n)$ with $n=1000$. There are loops of all sizes up to $O(\log (n))$, the order of its diameter. (Only topological relationships or connectivity between the nodes are relevant in the figure.)

a graph, and thus, all node pairs have at least one shortest path, or geodesic, between them. We denote a geodesic between a pair of nodes $A$ and $B$ by $[A B]$, which, if necessary, may be regarded as just one of possibly many shortest paths between the said node pair. In a metric graph $G$, a triangle $A B C$ is called $\delta$-thin if each of the shortest paths $[A B],[B C]$, and $[C A]$ is contained within the $\delta$ neighborhoods of the other two. More specifically,

$$
[A B] \subseteq \mathcal{N}([B C], \delta) \cup \mathcal{N}([C A], \delta),
$$

and similarly for $[B C]$ and $[C A]$. A triangle $A B C$ is $\delta$-fat if $\delta$ is the smallest $\delta$ for which $A B C$ is $\delta$-thin. The notion of (coarse) Gromov hyperbolicity is then defined as follows.

Definition 2.1. A metric graph is $\delta$-hyperbolic if all geodesic triangles are $\delta$-thin, for some fixed nonnegative $\delta$.

It is clear that all tree graphs are $\delta$-hyperbolic with $\delta=0$. We also observe that all finite graphs are $\delta$-hyperbolic for large enough $\delta$, e.g., by letting $\delta$ be equal to the diameter of the graph. Thus, the notion of coarse hyperbolicity is meaningful and nontrivial for infinite graphs. ${ }^{3}$

With these clarifications, we make the following observations. First, random graphs in the $p=c / n$ (middle) regime are not $\delta$-hyperbolic in the sense that they contain $\delta$-fat triangles for an arbitrary large $\delta$ a.s as $n \rightarrow \infty$. This is proved in Theorem 2.3. This observation was made experimentally in [16, see the taxonomy chart] and we provide a proof in this work. Figure 1 provides a visualization of this claim. Second, simulations suggest that the proportion of $\delta$-fat triangles is not only positive but is, in fact, quite significant for any $\delta$ as $n$ grows. These are shown in Section 3.1.

${ }^{3}$ However, one may translate this notion intuitively for large-scale graphs by saying that such a graph is $\delta$-hyperbolic when the smallest such $\delta$ is significantly smaller than the diameter of the graph [12]. 
Definition 2.2. Let $\left\{G_{n}\right\}_{n=1}^{\infty}$ be a family of random graphs. We say that a property holds asymptotically a.s. if the probability $p_{n}$ of this to occur goes to 1 as $n \rightarrow \infty$.

Theorem 2.3. For every non-negative $\delta$, the largest component of $G(n, c / n)$ with $c>1$ is not $\delta$-hyperbolic asymptotically, a.s.

Proof. Let $G=G(n, c / n)$ be an Erdös-Renyi random graph with $c>1$, and let $H$ be its largest connected component. It is well known that $H$ has $\gamma(c) n$ nodes asymptotically a.s. where $\gamma(c)$ is a function of $c$ and independent of $n$; see [4] for a proof of this result. Take $\rho>0$ and let us expose or highlight $(1-\rho) n$ of the nodes of $G$ chosen at random and the subgraph induced by these nodes. The remaining set of $\rho n$ nodes is called the hidden set and is denoted by $\mathcal{H}$. The exposed subgraph is just like an Erdos-Renyi random graph constructed on the exposed nodes, with $p=c / n=c(1-\rho) /(n(1-\rho))$. Since there are $n(1-\rho)$ nodes in the exposed subgraph, we can conclude that it has a giant component asymptotically, a.s., if $c(1-\rho)>1$, i.e., $0<\rho<\frac{c-1}{c}$. Furthermore, this giant component has size $(1-\rho) n \gamma((1-\rho) c)$. This giant component is contained in a single component of the original graph $G$. Because all the components of $G$ except its giant component have $O(\log (n))$ nodes, the giant component of the exposed subgraph is contained in the giant component of $G$.

Let $v$ be a node in $\mathcal{H}$ and let $k$ be a positive integer. The probability that $v$ has only two neighbors in $\mathcal{H}$ and no other neighbor is equal to

$$
(\rho n-1)(\rho n-2) p^{2}(1-p)^{n-3} / 2=\frac{1}{2} \rho^{2} c^{2} e^{-c}[1+O(1 / n)] .
$$

In Figure 2, these nodes are denoted 1 and $1^{\prime}$. The probability that nodes 1 and $1^{\prime}$ each have another unique neighbor in $\mathcal{H}$, which we call nodes 2 and $2^{\prime}$, is similarly

$$
(\rho n-3)(\rho n-4) p^{2}(1-p)^{2 n-8}=\rho^{2} c^{2} e^{-2 c}[1+O(1 / n)] .
$$

Repeating this argument, the probability that the nodes $(k-2)$ and $(k-2)^{\prime}$ are linked to nodes $(k-1)$ and $(k-1)^{\prime}$ is

$$
(\rho n-2 k+3)(\rho n-2 k+2) p^{2}(1-p)^{2 n-4 k+4}=\rho^{2} c^{2} e^{-2 c}[1+O(k / n)] .
$$

(We write $O(k / n)$ instead of writing $k O(1 / n)$ and absorbing the $k$ in the $O$, because we will soon choose $k$ to depend on $n$.) Finally, the probability that the nodes $(k-1)$ and $(k-1)^{\prime}$ are connected to nodes $k$ and $k^{\prime}$ in the giant component of the exposed set is

$$
\begin{array}{r}
n(1-\rho) \gamma((1-\rho) c)[n(1-\rho) \gamma((1-\rho) c)-1] p^{2}(1-p)^{2 n-4 k} \\
=c^{2}(1-\rho)^{2} \gamma^{2}((1-\rho) c) e^{-2 c}[1+O(k / n)] .
\end{array}
$$

Multiplying all these factors, the probability that $v$ is connected to the rest of the graph as shown in Figure 2 is

$$
p_{k}=\frac{\rho^{2 k-2}\left(c e^{-c}\right)^{2 k} \gamma((1-\rho) c)^{2}(1-\rho)^{2}}{2 e^{-c}}\left[1+O\left(k^{2} / n\right)\right] .
$$

When a node $v$ is connected to the rest of the graph in this manner, we say that $v$ is the base of a $k$-handle. 


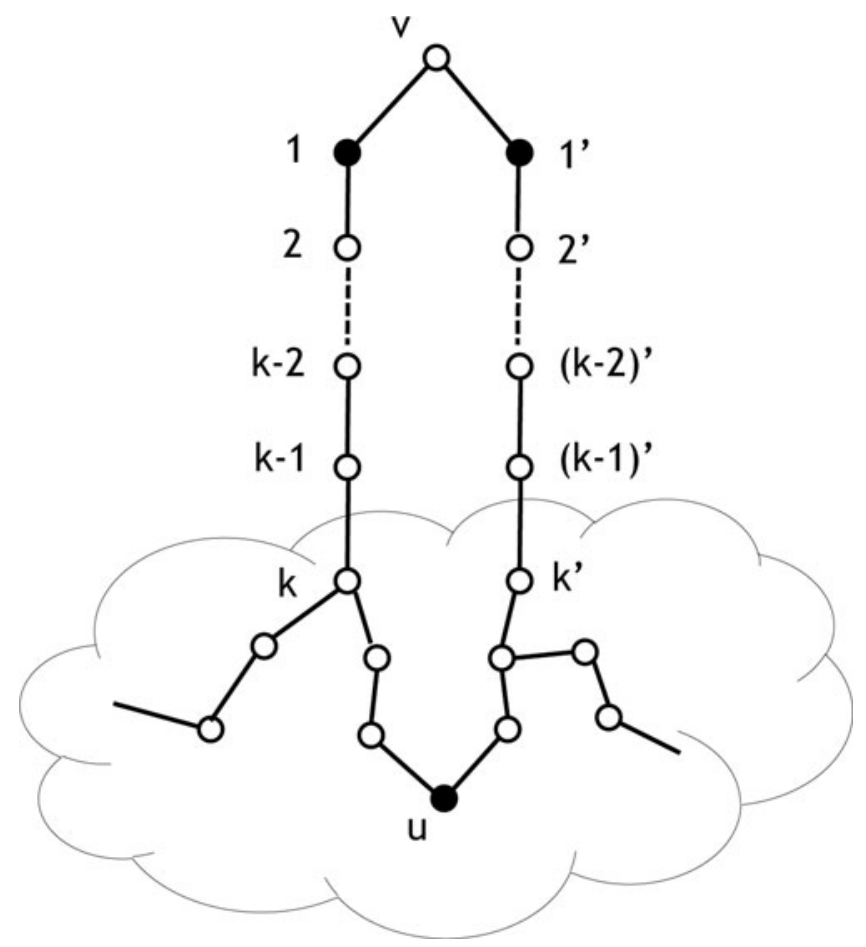

Figure 2 Depiction of a $k$-handle based at the point $v$. The nodes $k$ and $k^{\prime}$ are the nodes in the $k$-handle that belong to the giant component of the exposed set. The node $u$ is the midpoint of the geodesic path connecting $k$ and $k^{\prime}$ within the giant component of the exposed subgraph, which by definition does not include the node $v$.

Let $k$ and $k^{\prime}$ be the nodes in the $k$-handle that belong to the giant component of the exposed set. Note that since the nodes $k$ and $k^{\prime}$ are in the giant component of the exposed set, there exists at least one shortest path connecting them inside the exposed set and not passing through the node $v$. Let 1 and $1^{\prime}$ be the neighbors of $v$ (marked in black in Figure 2). Let $u$ be the midpoint of the geodesic path connecting $k$ and $k^{\prime}$ within the giant component of the exposed subgraph, which by definition does not contain $v$. Then, the sides of the geodesic triangle $\Delta\left(11^{\prime} u\right)$ are $1-v-1^{\prime} ; 1^{\prime}-k^{\prime}-u$ and $u-k-1$; and it is easy to see that this (loop) triangle is at least $\lfloor k / 2\rfloor$-fat, since all the edges of the side $1-2-\ldots-k-\ldots-u$ need to be covered from 1 (which is on $1^{\prime}-v-1$ ) and from $u$ (which is on $1^{\prime}-k^{\prime}-u$ ). (If the geodesic path connecting $k$ and $k^{\prime}$ has an even number of nodes, $u$ can be chosen to be either of the two nodes in the middle of $k-k^{\prime}$. Since $k-u$ and $k^{\prime}-u$ differ by only 1 , and $1-v-1^{\prime}$ has length 2 , the geodesics connecting 1 and $1^{\prime}$ to $u$ are still $1-k-u$ and $1^{\prime}-k^{\prime}-u$, and all the aforementioned subsequent steps remain valid.)

Let $X_{v}$ be the random variable that is equal to 1 if the node $v$ is the base of a $k$-handle with $v \in \mathcal{H}$ and 0 otherwise. As we have just seen, when $X_{v}=1$, there exists at least one $\lfloor k / 2\rfloor$-fat triangle in the graph. Define $Y_{k}$ to be the random variable $Y_{k}=\sum_{v \in \mathcal{H}} X_{v}$. To prove the existence of a $\lfloor k / 2\rfloor$-fat triangle in the giant component a.s., it is enough to prove that $\mathbb{P}\left(Y_{k} \geq 1\right) \rightarrow 1$ as $n \rightarrow \infty$. We will prove the following stronger result: for every constant $0<t<(2(c-\log (\rho)))^{-1}$, there are a.s. $(t \log (n))$-handles as $n \rightarrow \infty$. Taking 
$k=t \log (n)$ in (2.3) we obtain

$$
\begin{aligned}
p_{t \log (n)} & =\left(\frac{\gamma((1-\rho) c)^{2}(1-\rho)^{2} e^{c}}{2 \rho^{2}}\right)\left(\rho c e^{-c}\right)^{2 t \log (n)}\left[1+O\left(\log ^{2}(n) / n\right)\right] \\
& =\left(\frac{\gamma((1-\rho) c)^{2}(1-\rho)^{2} e^{c}}{2 \rho^{2}}\right) n^{2 t \log \left(\rho c e^{-c}\right)}\left[1+O\left(\log ^{2}(n) / n\right)\right] .
\end{aligned}
$$

Let us define

$$
\theta(c):=\left(\frac{\gamma((1-\rho) c)^{2}(1-\rho)^{2} e^{c}}{2 \rho^{2}}\right)
$$

Then, the expected number of $(t \log (n))$-handles in $\mathcal{H}$ is

$$
\begin{aligned}
\mathbb{E}\left(Y_{t \log (n)}\right) & =\sum_{v \in \mathcal{H}} \mathbb{E}\left(X_{v}\right)=\rho n p_{t \log (n)}=\rho \theta(c) n^{1+2 t \log \left(\rho c e^{-c}\right)}\left[1+O\left(\log ^{2}(n) / n\right)\right], \\
& =\rho \theta(c) n^{1+2 t \log (c)-2 t \log \left(e^{c} / \rho\right)}\left[1+O\left(\log ^{2}(n) / n\right)\right]
\end{aligned}
$$

since the random variables $X_{v}$ are identically distributed and Bernoulli. Because by assumption $0<t<(2(c-\log (\rho)))^{-1}$, we see that this quantity goes to infinity as $n$ increases. Furthermore, since $\rho<(c-1) / c$, we know that $\rho c e^{-c}<(c-1) e^{-c}<1$, and therefore, from (2.4), we see that $p_{t \log (n)}$ goes to zero as $n$ increases.

We now calculate the variance of $Y_{t \log (n)}$. It is easy to see that

$$
\mathbb{V}\left(Y_{t \log (n)}\right)=\rho n p_{t \log (n)}\left(1-p_{t \log (n)}\right)+\sum_{v \in \mathcal{H}} \sum_{w \neq v \in \mathcal{H}}\left[\mathbb{E}\left(X_{v} X_{w}\right)-p_{t \log (n)}^{2}\right]
$$

To obtain $\mathbb{E}\left(X_{v} X_{w}\right)$, we note that this is zero unless $X_{v}=X_{w}=1$, i.e., $v$ and $w$ are both at the bases of $t \log (n)$-handles. The conditional probability that $X_{w}=1$ when $X_{v}=1$ is zero if $w$ is any of the $2 t \log (n)-2$ nodes in $\mathcal{H}-v$ that are in the $t \log (n)$ handle whose base is $v$. If $w$ is any of the remaining $n \rho-2 t \log (n)+1$ nodes in $\mathcal{H}-v$, the probability that $X_{w}=1$ can be obtained by a process similar to the derivation of (2.3), with slight adjustments. For example, the probability that $w$ has only two neighbors in $\mathcal{H}$ and no other neighbor is equal to

$(\rho n-2 t \log (n))(\rho n-2 t \log (n)-1) p^{2}(1-p)^{n-2 t \log (n)-2} / 2=\frac{1}{2} \rho^{2} c^{2} e^{-c}[1+O(t \log (n) / n)]$.

The other factors that go into $p_{k}$ can be calculated similarly. Combining all of them, the probability that $X_{w}=1$ is equal to $p_{t \log (n)}\left[1+O\left(t^{2} \log ^{2}(n) / n\right)\right]$. Therefore,

$$
\begin{aligned}
\mathbb{V}\left(Y_{t \log (n)}\right) & =\rho n p_{t \log (n)}\left(1-p_{t \log (n)}\right) \\
& +\rho n(\rho n-2 t \log (n)+1) p_{t \log (n)}^{2} O\left(t^{2} \log ^{2}(n) / n\right) \\
& -\rho n(2 t \log (n)-2) p_{t \log (n)}^{2} .
\end{aligned}
$$

Combining the results for $\mathbb{E}\left(Y_{t \log (n)}\right)$ and $\mathbb{V}\left(Y_{t \log (n)}\right)$,

$$
\frac{\mathbb{V}\left(Y_{t \log (n)}\right)}{\mathbb{E}\left(Y_{t \log (n)}\right)^{2}}=\frac{\left(1-p_{t \log (n)}\right)}{\rho n p_{t \log (n)}}+\left[1-\frac{2 t \log (n)-1}{\rho n}\right] O\left(t^{2} \log ^{2}(n) / n\right)-\frac{2 t \log (n)-2}{\rho n} .
$$


It is easy to see that the last two terms in the sum tend to zero as $n \rightarrow \infty$. By our election of $t$, we know that $p_{t \log (n)} \rightarrow 0$ and that $n p_{t \log (n)} \rightarrow \infty$ as $n \rightarrow \infty$, so that

$$
\lim _{n \rightarrow \infty} \frac{\mathbb{V}\left(Y_{t \log (n)}\right)}{\mathbb{E}\left(Y_{t \log (n)}\right)^{2}}=0 .
$$

It is now straightforward to apply Chebyshev's inequality:

$$
\mathbb{V}\left(Y_{t \log (n)}\right) \geq \mathbb{P}\left(Y_{t \log (n)}=0\right) \mathbb{E}\left(Y_{t \log (n)}\right)^{2}
$$

to obtain

$$
\lim _{n \rightarrow \infty} \mathbb{P}\left(Y_{t \log (n)}=0\right)=0
$$

and our result follows.

As a corollary of the previous proof we have an alternative proof of the following wellknown result.

Corollary 2.4. The largest component of $G(n, c / n)$ with $c>1$ has no spectral gap asymptotically a.s.

This result follows because the previously constructed $(t \log (n))$-handles are cut sets with $2 t \log (n)+1$ nodes and only two boundary nodes.

\section{SIMULATIONS}

\subsection{Numerical Results on Percentage of Fat Triangles}

We have seen that random graphs $G\left(n, p_{n}\right)$ in the regime $p_{n}=c / n$ are nonhyperbolic asymptotically a.s. However, these random graphs appear to be non-hyperbolic in a much stronger sense. To see how, consider the chart in Figure 3. This is an example of a curvature plot; see [16]. For any triangle $\Delta=A B C$, the corresponding $\delta_{\Delta}$ is defined by

$$
\delta_{\Delta}=\min _{D} \max \{d(D ; A B), d(D ; B C), d(D ; A C)\}
$$

where $d(D ; A B)$ is the distance between $D$ and the node on $A B$ that it is closest to. It can be shown see [3] that the maximum of $\delta_{\Delta}$ over all possible triangles in a graph is finite if and only if the graph is $\delta$-hyperbolic. Instead of the maximum, Figure 3 shows the average value of $\delta_{\Delta}$ for all triangles whose shortest side is $l$, as a function of $l$. Results for random graphs of various sizes, with $p=2 / n$ are shown. The results show a linear increase in $\delta_{a}(l)$ saturating at a plateau whose height increases as the size of the graph is increased. The same results are rescaled in the right panel, where all the curves are shifted down and to the left by amounts proportional to $\ln n$. Thus, the plot shows $\delta_{a}(l)-c_{1} \ln n$ versus $l-c_{2} \ln n$, where $c_{1}$ and $c_{2}$ are adjusted to achieve the best possible fit. As shown in the figure, except the leftmost part of each curve where $l \sim O(1)$, all the curves for different values of $n$ collapse onto a single universal curve. This, together with the fact that the curves in the left panel also coincide before their plateaus, implies that the rising part of the universal curve is linear. If $n \rightarrow \infty$, any finite $l \gg 1$ is on the rising part of the universal curve, and therefore, $\delta_{a}(l)$ increases linearly with $l$, with the plateau pushed out to $l \rightarrow \infty$.

Thus, we see that a significant fraction of triangles in a typical instantiation of $G(n, c / n)$ are $\delta$-fat, a stronger demonstration of non-hyperbolicity. Therefore, it seems that 


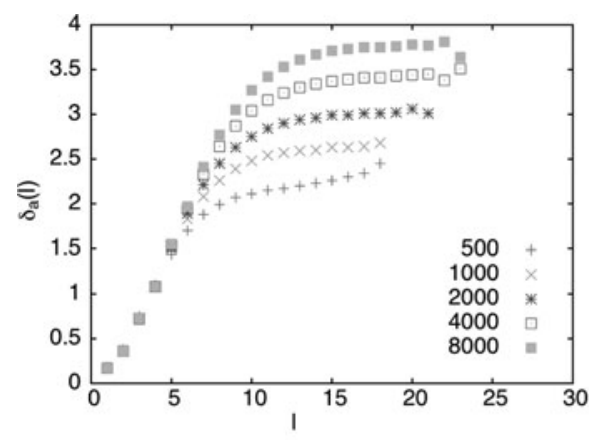

(a)

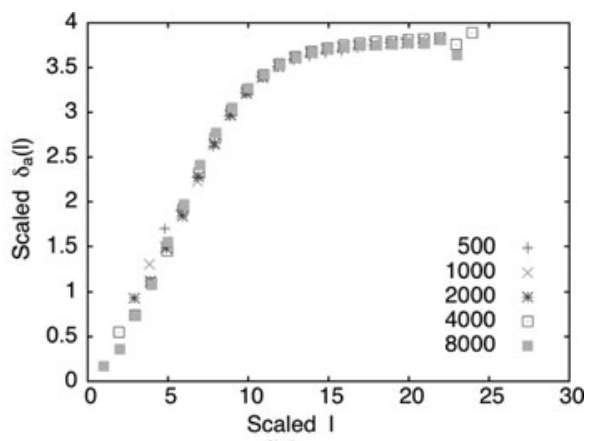

(b)

Figure 3 Curvature plot for random graphs with $p=2 / n$ and various values of $n$. Only the giant component of each graph was retained, and an average over many randomly chosen triangles in 40 instantiations of the graph was performed; (b) shows the same curves as those in (a) but shifted down and to the left by amounts proportional to $\ln (n / 8000)$.

$\delta$-fat triangles not only exist a.s., but they are abundant! Even though we do not yet have direct proof of this observation, Figure 3 clearly shows the predominance of fat triangles in $G(n, c / n)$ due to the increasing (average) $\delta$. Thus, $G(n, c / n)$ random graphs are far from hyperbolic.

Figure 4 shows that if the average $\delta_{\Delta}$ for all triangles with the same longest side $l_{\max }$ is plotted as a function of $l_{\max }$, as $n$ increases, the height of the curves decreases for small $l_{\max }$ and increases for large $l_{\max }$, with the boundary between the two regions shifting to the right as $n$ increases. Thus, $\lim _{n \rightarrow \infty} \delta_{a}\left(l_{\max }\right)=0$ for any fixed $l_{\max }$, in accordance with the local treelike structure. Similar results are seen for $p=3 / n$ in Figure 5.

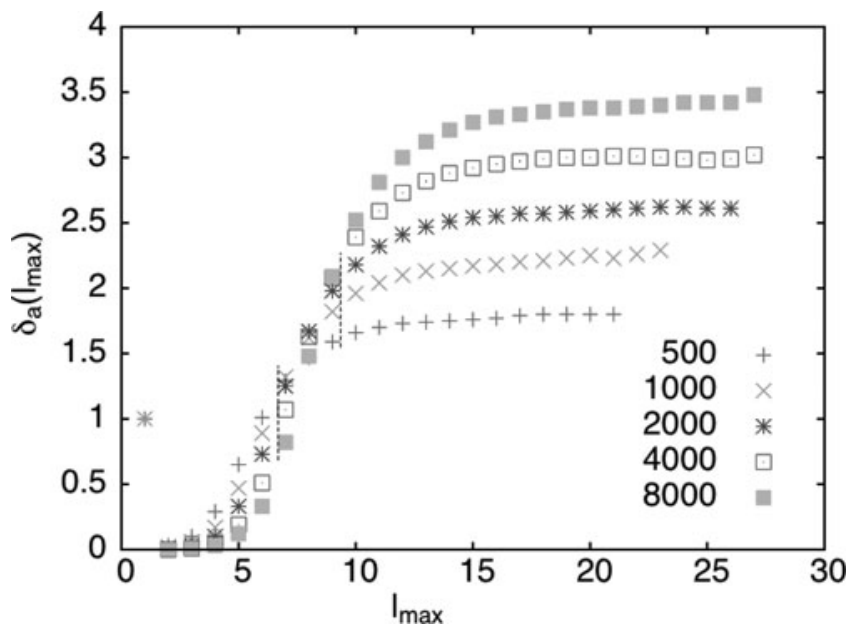

Figure 4 Average $\delta_{\Delta}$ for all triangles with longest side $l_{\max }$ as a function of $l_{\max }$, for various graph sizes. The points in the region between the dashed lines show that, as $n$ is increased, the range of $l_{\max }$ over which the curves move downward expands to the right. 

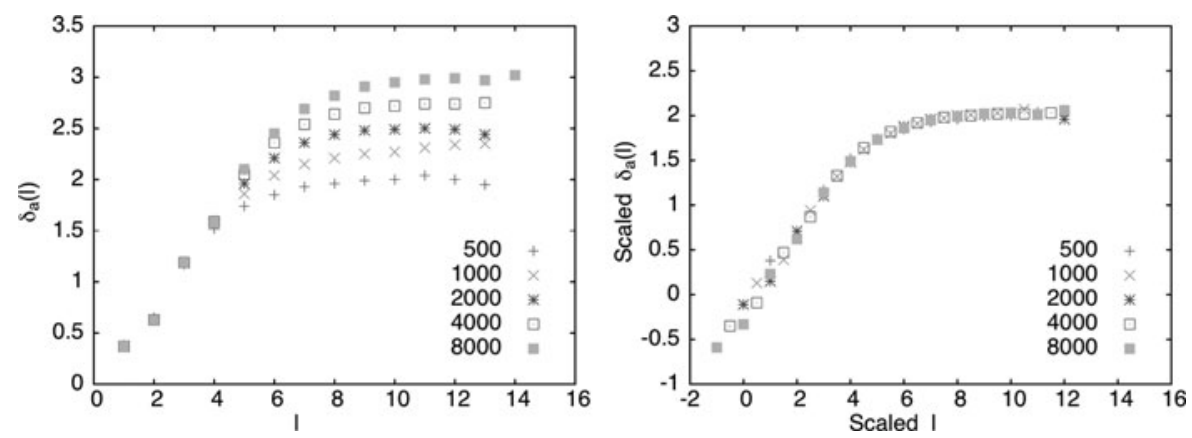

Figure 5 Plots for random graphs with $p=3 / n$, similar to Figure 3 for $p=2 / n$.

\subsection{Some Simulation Results on the Bulk Region of the Spectrum}

Here we present some simulations of the spectral measure $\mu_{n}$ for the Laplacian of the Erdös-Renyi graphs $G(n, c / n)$. It is known that these measures converge weakly to a probability measure $\mu_{\infty}$ [2]. Observe in Figures 6 and 7 how close these probabilities are in the bulk region to the McKay probability measure, the spectral measure of the Laplacian of the $c$-regular tree, which is given by [14] to be

$$
\mu(d x)=\frac{\sqrt{4(c-1)-c^{2}(1-x)^{2}}}{2 \pi c\left(1-(1-x)^{2}\right)} \cdot \mathbf{1}_{\left[1-\frac{2 \sqrt{c-1}}{c}, 1+\frac{2 \sqrt{c-1}}{c}\right]} .
$$

It is interesting to compare these plots with the spectral measure of the finite truncated tree. To be more precise, fix $c$ and let $T_{c}$ be the infinite regular tree of degree $c$. The spectral measure $v$ for the Laplacian of this tree is given by (3.2). Consider now, for each finite $n$, the spectral measure $v_{n}$ of the truncated finite tree is constructed from $T_{c}$ by keeping only the first $n$ generations. It is a well-known result see [11] that these measures do converge to a
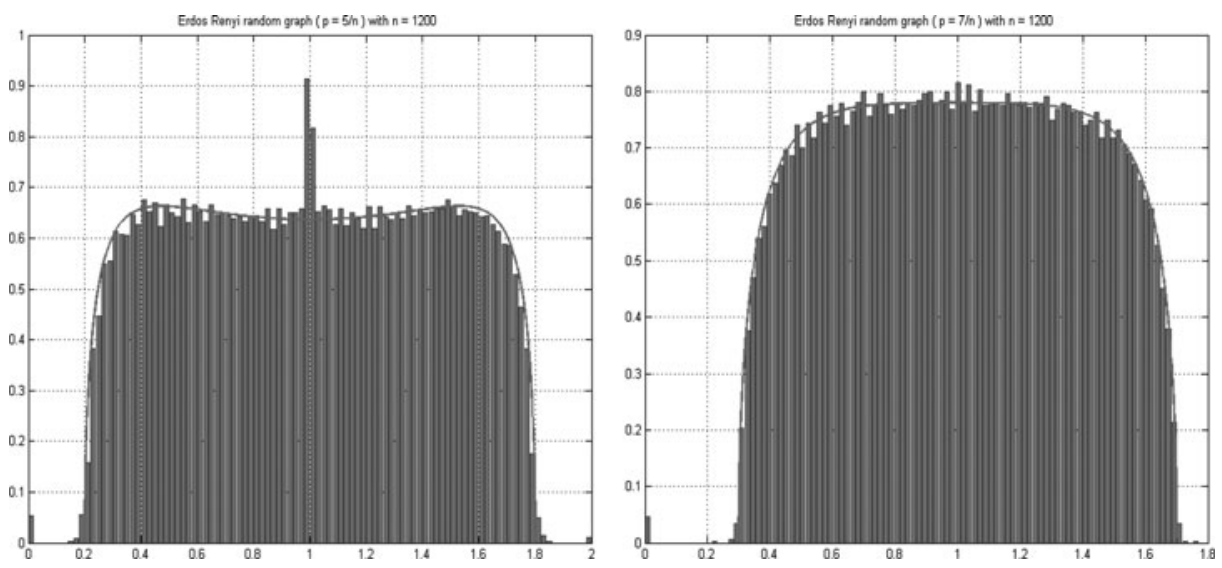

Figure 6 The left chart in grey is $\mu_{n}$, the spectral density of $G(n, 5 / n)$ for $n=1200$. The right chart is $\mu_{n}$, the spectral density of $G(n, 7 / n)$ for $n=1200$. The curves are the McKay densities, the spectrum of the infinite regular tree with degrees $c=5$ and $c=7$, respectively. 

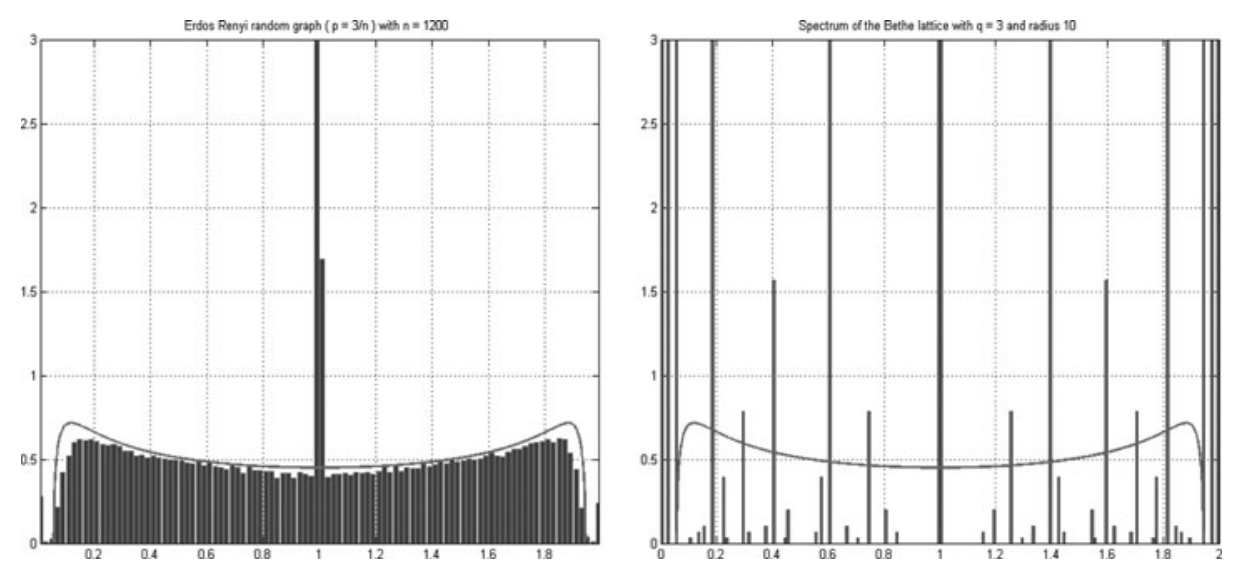

Figure 7 The left chart in grey is $\mu_{n}$, the spectral density of $G(n, 3 / n)$ for $n=1200$. The curve is the McKay density, the spectral density of the infinite regular tree, with degree $c=3$. In the right chart, in grey is $v_{n}$, the spectral density of the truncated tree with degree $c=3$ and radius 10. The curve is the McKay density of degree $c=3$.

measure $v_{\infty}$. However, $v_{\infty}$ and $v$ are very different. For instance, the measure $v_{\infty}$ has atoms, whereas $v$ does not. This is because repeated eigenvalues occur with large multiplicities. The main heuristic reason for this phenomenon is that the truncated tree has a large number of nodes with degree 1, creating a significant boundary effect. See Figure 7 to see this phenomenon.

These figures clearly show that the distribution of the spectrum of large ErdösRenyi random graphs provides a better approximation for the spectral measure of the corresponding infinite regular tree in the bulk region than do large finite truncated trees of the same degree. We do not yet have a complete explanation for this. We note that this result is in contrast to the regime $n p_{n} \rightarrow \infty$ in which the distribution of the eigenvalues follows the well-known semicircle law [5].

Of course, the two spectral measures $\mu_{\infty}$ and $\nu_{\infty}$ are not exactly the same; we have shown already that $v_{\infty}$ does not have a spectral gap, whereas $\mu_{\infty}$ does. We also observe in Figure 6 that the measure $\mu_{n}$ for small values of $c$ seems to spike at 1 . As seen in Figure 7, the size of this spike seems to decrease as $c$ increases, but we do not know if the spike disappears as $n \rightarrow \infty$ for fixed small $c$. Nevertheless, the close similarity observed between $\mu_{\infty}$ and $\nu_{\infty}$ provides an approximate expression for $\mu_{\infty}$. The question remains whether an exact expression can be obtained for $\mu_{\infty}$; more generally, if we consider the branching process generated by any probability distribution in the natural numbers $\mathbb{N}$, whether an expression can be obtained for the spectral measure of the normalized Laplacian for this graph.

\section{FUNDING}

This work was supported by AFOSR Grant Numbers FA9550-08-1-0064 (for O. N., as a consultant with Bell Labs) and FA9550-11-1-0278. 


\section{REFERENCES}

1. I. Benjamini, G. Kozman, N. Wormald. "The Mixing Time Of The Giant Component Of A Random Graph.” Available at http://arxiv.org/pdf/math/0610459v1.pdf (2006).

2. C. Bordenave and M. Lelarge. "Resolvent of Large Random Graphs." Rand. Struct. Alg. 37:3 (2010), 332-352.

3. M. Bridson and A. Haeiger. Metric Spaces of Non-Positive Curvature, Grundlehren der mathematischen Wissenschaften, Vol. 319. Berlin: Springer-Verlag, 1991.

4. F. Chung. Spectral Graph Theory, CBMS Regional Conference Series in Mathematics 92. Providence, RI: American Mathematical Society, 1994.

5. F. Chung, L. Liu, and V. Vu. "Spectra of Random Graphs with Given Expected Degrees." Proc. Natl. Acad. Sci. USA 100:11 (2007), 6313-6318.

6. A. Coja-Oghlan and A. Lanka. "The Spectral Gap of Random Graphs with Given Expected Degrees." Elect. J. Combinatorics 16:1 (2009), R138.

7. P. Erdös and A. Renyi. "Random Graphs I." Publ. Math. Inst. Hungar. Acad. Sci. 6 (1959), 290-297.

8. N. Fountoulakis and B. A. Reed. "The Evolution of the Mixing Rate of a Simple Random Walk on the Giant Component of a Random Graph.” Rand. Struct. Alg. 33:1 (2008), 68-86.

9. E. Gilbert. "Random Graphs." Ann. Math. Stat. 30:4 (1959), 1141-1144.

10. M. Gromov. "Hyperbolic Groups.” In Essays in Group Theory, pp. 75-263, New York: Springer, 1987.

11. L. He, X. Liu, and G. Strang. "Trees with Cantor Eigenvalue Distribution." Studies Appl. Math. 110:2 (2003), 123-136.

12. E. Jonckheere and P. Lohsoonthorn. "Geometry of Network Security." Proc. American Control Conf. 2 (2004), 976-981.

13. B. Karrer and M. E. Newman. "Random Graphs Containing Arbitrary Distribution of Subgraphs." Phys. Rev. E 82 (2010), 066118.

14. B. McKay. “The Expected Eigenvalue Distribution of a Large Regular Graph.” Lin. Alg. Appl. 40 (1981), 203-216.

15. M. Mezard and A. Montanari. Information, Physics and Computation. Oxford: Oxford University Press, 2009.

16. O. Narayan and I. Saniee. "Large-Scale Curvature of Networks.” Phys. Rev. E 84 (2011), 066108. Available at http://arxiv.org/abs/0907.1478. 